\title{
Exploring Touching Learning Environments
}

\author{
Gustavo Ramírez González, Mario Muñoz Organero and Carlos Delgado Kloos \\ Carlos III University of Madrid, Leganes Campus, Madrid, Spain. \\ \{gramirez@inv.it.uc3m.es, munozm@it.uc3m.es, cdk@it.uc3m.es\}
}

\begin{abstract}
There are several ways for learning, but in actual society, the proliferation of technology alternatives enables multiple learning scenarios and environments. Part of the most important technologies for this, are associated to mobile phones. Classically, the mobile were expected to deliver content, but they were not specifically designed for interacting with real objects and context. This paper proposes the concept of Touching Learning Environments as part of the evolution of M-learning and Ubiquitous Computing, enabling touching technologies in mobile devices; this technology is known as Near Field Communication NFC.
\end{abstract}

\section{Introduction}

The actual deployment and evolution of ubiquitous computing is possible due the progress and widespread dissemination of some enabler technologies such as RFID, Bluetooth and NFC. The use of these technologies combined with the extensive market penetration of mobile phones and their powerful options for personalization and mobility, offer an unprecedented atmosphere for the creation of new learning environments.

This new learning environment consists basically in the interaction of a person with the context, basically by touching things, this things will give information and it could be part of learning activities. The long term vision is a place where people can get information and learning experience by "talking" or manipulating objects. The goal of this paper is to definite this concept and show some possible scenarios. To facilitate the reading, most of technical details will eliminate, but a basic explanation of technologies involved will be included.

The structure of the paper is as follows. First some background about related technology will be exposed in section 2 . Then a set of generic touching learning 
scenarios are exposed in section 3. Finally some conclusions and future work are presented at the end of the paper.

\section{Enabling technologies for Touching Learning}

\subsection{RFID Radio Frequency Identification}

RFID [1] is probably the most relevant technology in the building of the ubiquitous computing. It basically began as a promise to eliminate many business problems especially in the logistics of units, material handling and inventory in general for the supply chain and the retail sector. Furthermore, other applications [2] such as access control, luggage tracking, electronic payment systems, homeland security, livestock history, library tracking of books and receiving assistance. On the other hand there are some interesting experiences motivated by the every day activities of people or explore the concept of smart products.

\subsection{NFC Near Field Communication}

Near Field Communication (NFC) is one of the latest wireless networking technologies based on RFID. As a short-range wireless connectivity technology, NFC provides intuitive, simple, and safe communication between electronic devices. NFC [3] is distinguished by its intuitive interface. Different trials of this technology have successfully illustrated how people carrying mobile phones can make purchases, get directions, exchange information, and buy transportation tickets simply by bringing them close to NFC-enabled devices embedded in information kiosks, retail, advertising, vending machines, and other devices. Formed in 2004, the NFC Forum [4] envisions a world where people can access content and services in an intuitive way, leading to secure universal commerce and connectivity. The Forum believes that NFC technology makes business sense for transportation and content providers, device manufacturers, financial services organizations, and more. An ABI Research forecasts that by 2012, some 292 million handsets (over 20 percent of the global mobile handset market) will ship with built in NFC capabilities [5].

\section{Generic Touching Learning Scenarios}

At this stage, "Touching Learning" or "Learning by Touching" can be defined as: the used services and applications in a learning environment where the learning actor can interact with environment resources for learning purposes or for communication, only by touching.

NFC, as technological enabler can be used in several ways. A set of generic learning touching scenarios are presented. These scenarios are expected to be as generic as possible to be applied in any specific instances. The first one describes the basic function of "Touching for searching". The second presents a "Personal 
Physical Context", this is the relationship established between a person and several objects. The third one shows the action of direct interaction and control from the mobile user to the surrounding "intelligent" objects. Some implementations of this touching learning scenarios can be found in [6].

\subsection{Touching to Search in the Physical Context}

The Web is a huge repository of information that must be organized in order to be able to find useful things for the final user. Nowadays there is a well known series of search engines. Due to their friendly web based interfaces it is easy to find information in the Web. In general, there are various mechanisms to do that, but the most popular is based on references and indexation. By introducing these mechanisms, the concept of searching can define. The main difference between the information in the Web and the information in the Physical context is that the information is associated and lives attached to a particular object, describing it and providing some times the access mechanisms for its remote control and adaptation. In Figure 1 show an application of this scenario in a lab, where student look for the content of the cabinet touching the tag and it display a set of codes or names related with the content.
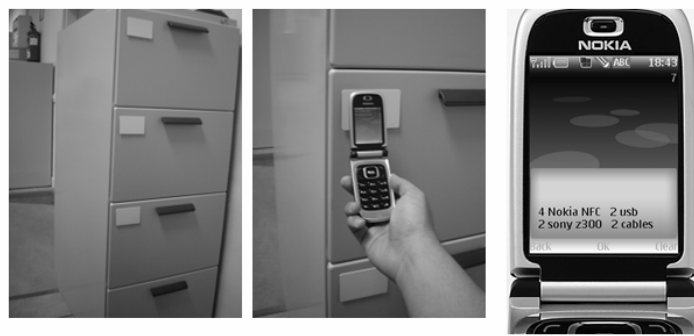

Figure 1. Scenario of Touching to Search.

\subsection{Touching in Personal Physical Context}

Touching in personal Physical Context is the relationship established between a person and several objects that personalizes the behavior of the objects according to the mobile user preferences. Mobile users with an NFC enabled mobile device can personalize the behavior of their local environment simply by touching the objects, exchanging profiles and taking the appropriate actions. A person in its daily routine interacts with diverse physical objects. This interaction in an NFC enable scenario is produced by the contact of mobile devices with physical objects with different purposes. Due to this relation, the information obtained by the physical contact with objects can be stored, analyzed and processed in the mobile device or can be shared and synchronized with other personal devices or with external computers depending on the information in the user profile. 


\subsection{Touching to Adapt Physical Context}

This third scenario establishes an association between a user and the surrounding objects which is by itself active. The physical environment of the user, adapt to the user not only in an automatic process which is the result of the exchange and processing of user and object profiles, but in a proactive way which can be controlled by the user by means of a peer to peer communication process. Using NFC P2P capabilities allows us to implement real scenarios implementing this idea. As an example we can find a classroom containing some devices such as an overhead projector, a bookshelf and some other devices which can be controlled by the professor or the students in some different ways depending on their profiles.

\section{Conclusions and future work}

This paper proposes the concept of Touching Learning Scenarios using NFC as technology to enable mobility and interaction with physical spaces. It also proposes three generic scenarios. Mobile Phone is one of the best candidates to enable transparency in learning systems. This basic technology combined with others as NFC, increases the possibilities of adoption. As part of our future work, more scenarios are expected specially using other characteristics of NFC more than basic tagging. The design of experiences linking the Touching Learning concept with formal and informal learning is expected.

\section{Acknowledgments}

This work was supported by the Spanish "Programa Nacional de Tecnologías de la Sociedad de la Información" through the projects TSI2005-08225-C07-01 and -02. Gustavo Ramirez is funded by the EU Programme Alban, scholarship No. E06D101768CO and by University of Cauca.

\section{References}

1. P. Sweeney, RFID For Dummies, Wiley Publishing, 2005.

2. RFID Journal web site; http://www.rfidjournal.com/

3. NFC Forum. White Paper, The Keys to Truly Interoperable Communications. 2006.

4. NFC Forum http://www.nfc-forum.org

5. ABI Research Press Release, Twenty Percent of Mobile Handsets Will Include Near Field Communication by 2012.

6. G. Ramírez, M. Muñoz and C. Delgado, IOT early possibilities in learning scenarios, Workshop on Designing the Internet of Things for Workplace Realities: Social and Cultural Aspects in Design and Organization (Social-IoT), Zurich, Switzerland, March 26, 2008. 